\title{
Efeito da salinidade e modo de aplicação da água de irrigação no crescimento e produção de alho(1)
}

\author{
Júlio Roberto de Araújo Amorim( ${ }^{(2)}$, Pedro Dantas Fernandes( ${ }^{(3)}$, Hans Raj Gheyi(4) e Norma César de Azevedo(3)
}

\begin{abstract}
Resumo - Na Paraíba é comum a irrigação do alho por aspersão utilizando águas salinas, o que pode causar sérios danos às plantas. Este trabalho, realizado em casa de vegetação, teve por objetivo avaliar os efeitos sobre crescimento e produção do alho a partir de dois modos de aplicação de água, molhando ou não a folhagem das plantas, combinados com cinco níveis de salinidade da água de irrigação, que variaram entre 0,6 e 3,0 dS m$~ m^{-1}$. O desenvolvimento das plantas foi avaliado aos 30, 60, 90 e 120 dias após o plantio (DAP). As plantas de alho foram relativamente tolerantes à salinidade na brotação de bulbilhos e crescimento até 30 DAP. No final do ciclo (90-120 DAP), a parte aérea da planta e a formação dos bulbos foram afetadas pelo molhamento da folhagem das plantas. A salinidade começou a afetar a parte aérea das plantas entre 30-60 dias, enquanto o bulbo passou a ser afetado entre 60-90 DAP. A fase final de formação do bulbo (últimos 30 dias do ciclo) foi a mais sensível à salinidade. A razão bulbar não pode ser utilizada como parâmetro de avaliação de tolerância do alho à salinidade.
\end{abstract}

Termos para indexação: Allium sativum, qualidade de água, fitomassa, bulbo.

Effect of irrigation water salinity and its mode of application on garlic growth and production

Abstract - In Paraíba State, Brazil, garlic is usually irrigated by sprinkler system using waters of varying salt concentrations that may cause damage to plants. The present study was carried out under greenhouse conditions testing five levels of water salinity varying from 0.6 to $3.0 \mathrm{dS} \mathrm{m}^{-1}$ and two modes of water application, wetting or not plant leaves. The growth and development of plant was evaluated at 30,60, 90 and 120 days after planting (DAP). Garlic plants are relatively tolerant to salinity at bulb formation stage and initial growth up to 30 days. During the final stage (90-120 DAP), wetting of leaves affected more the growth of aerial parts and the number of garlic cloves. The salinity levels started affecting aerial parts during the period of 30-60 days while the bulb was affected only between 60-90 DAP. The most sensitive phase of bulb growth to salinity was the last 30 days of crop cycle. The bulb ratio can not be utilized as a characteristic for evaluation of salinity tolerance in garlic.

Index terms: Allium sativum, water quality, phytomass, bulb.

\section{Introdução}

A cultura do alho (Allium sativum) é importante não apenas por seu valor comercial, mas, também,

\footnotetext{
(1) Aceito para publicação em 26 de abril de 2001

(2) Embrapa-Centro de Pesquisa Agropecuária dos Tabuleiros Costeiros, Av. Beira Mar, 3250, Caixa Postal 44, CEP 49025-040 Aracajú, SE.

E-mail: jramorim@cpatc.embrapa.br

(3) Universidade Federal da Paraíba (UFPB), Centro de Ciências e Tecnologia (CCT), Dep. de Engenharia Agrícola, Caixa Postal 10087, CEP 58109-970 Campina Grande, PB. E-mail: pdantas@deag.ufpb.br, azevedo@deag.ufpb.br

(4) UFPB, CCT. Bolsista do CNPq. E-mail: hans@deag.ufpb.br
}

por suas propriedades medicinais. Todavia, a quantidade e qualidade da produção nacional de alho não atendem à crescente demanda e às exigências do mercado consumidor brasileiro, o que induz à importação do produto (Mueller \& Biasi, 1990). Nos últimos anos, com a geração de novas tecnologias, principalmente em Santa Catarina e Minas Gerais, onde se concentram as maiores áreas de cultivo e produtividade, vem aumentando a qualidade da produção nacional. A irrigação é uma das práticas mais importantes na exploração da cultura do alho, por sua alta exigência em níveis adequados de umidade do solo para atingir altas produções (Embrapa, 1997a).

Em várias regiões do Nordeste há condições propícias ao cultivo de alho, desde que sejam supridas 
as necessidades hídricas da cultura, através de irrigação, como vem ocorrendo no Município de Cabaceiras, na Paraíba.

Nessas áreas, é comum o uso de águas de cacimbas e de poços escavados no álveo dos rios, mediante irrigação por aspersão, molhando a folhagem. Essas águas nem sempre são adequadas para irrigação, contribuindo para o aparecimento de problemas de salinidade e, conseqüentemente, nutricionais, com prejuízos para os agricultores. A água de irrigação, mesmo de baixa salinidade, pode tornar-se um fator de salinização do solo, se não for manejada corretamente (Ayers \& Westcot, 1991; Lazof \& Bernstein, 1999).

$\mathrm{Na}$ literatura, são poucos os trabalhos sobre salinidade da água na cultura de alho. Magalhães (1986) e François (1994) citam ser esta compósita muito sensível ao íon cloreto, mesmo em níveis relativamente baixos. Ayers \& Westcot (1991) classificam o alho como sensível à salinidade sem, contudo, apresentar valores relacionando produção e níveis de condutividade elétrica.

O excesso de sódio afeta o diâmetro e o peso dos bulbos tornando a relação entre a porcentagem de sódio trocável (PST) e a produção negativa (Singh \& Abrol, 1985).

Conforme Mangal et al. (1990), dependendo da cultivar, pode haver queda de até $50 \%$ na produção de bulbos de alho, na faixa de condutividade elétrica do extrato de saturação do solo entre 5,60 e 7,80 dS m-1. Segundo esses autores, a produção declina 1,68\% para cada incremento unitário na condutividade elétrica do extrato de saturação (CEes) acima de $1,70 \mathrm{dS} \mathrm{m}^{-1}$. François (1994) observou redução de $50 \%$ na produção de alho com CEes de $7,3 \mathrm{dS} \mathrm{m}^{-1}$, com declínio de $14,3 \%$ por unidade de incremento da salinidade acima de $3,9 \mathrm{dS} \mathrm{m}^{-1}$.

O objetivo deste trabalho foi estudar o efeito de níveis de sais na água de irrigação e do modo de aplicação da água, molhando ou não a folhagem das plantas, sobre o crescimento e desenvolvimento do alho.

\section{Material e Métodos}

O trabalho foi realizado em casa de vegetação do Departamento de Engenharia Agrícola da Universidade Fede- ral da Paraíba, em Campina Grande, PB. O solo utilizado no experimento foi classificado como Podzólico Amarelo Equivalente Eutrófico, coletado em uma área cultivada, à profundidade de 0-30 cm, na Estação Experimental da Emepa, em Lagoa Seca, PB. Após ter sido secado ao ar, o solo foi destorroado, passado em peneira com malha de 2,0 mm e homogeneizado. Em seguida, misturou-se a esse material esterco de curral, curtido e peneirado, na proporção de $3,3 \%$, com base em peso.

$\mathrm{Na}$ Tabela 1 constam os dados de caracterização físicohídrica e química do solo, obtidos segundo Embrapa (1997b). No cultivo das plantas foram utilizados recipientes de plástico (bacias) com capacidade para $20 \mathrm{~kg}$ de solo (19 $\mathrm{cm}$ de altura por $38 \mathrm{~cm}$ de diâmetro).

Os tratamentos consistiram da combinação de níveis de salinidade da água de irrigação (NS) e modo de aplicação da água (MA). Os níveis de salinidade testados, em termos de condutividade elétrica da água de irrigação (CEa) foram: $0,6,1,2,1,8,2,4$ e $3,0 \mathrm{dS} \mathrm{m}^{-1}$; e os modos de aplicação foram: $\mathrm{MA}_{1}$ : aplicação da água no solo, sem molhar as folhas, e $\mathrm{MA}_{2}$ : aplicação da água através de um pequeno regador manual, molhando as folhas da planta. O delineamento experimental adotado foi inteiramente casualizado, com quatro repetições, em esquema fatorial $5 \times 2$. Cada parcela experimental constou de um vaso contendo de quatro a dez plantas, dependendo do estágio de avaliação, uniformemente distribuídas em cada recipiente.

Os níveis de sais utilizados nas irrigações foram obtidos a partir da diluição de uma solução estoque, previa-

Tabela 1. Características físico-hídricas e químicas do material do solo franco-arenoso utilizado no experimento

\begin{tabular}{lc}
\hline Atributos & Valores \\
\hline Densidade global $\left(\mathrm{kg} \mathrm{dm}^{-3}\right)$ & 1,47 \\
Retenção de umidade $\left(\mathrm{g} \mathrm{kg}^{-1}\right)$ & \\
$10 \mathrm{kPa}$ & 155,3 \\
$1500 \mathrm{kPa}$ & 73,2 \\
$\mathrm{pH}$ (pasta de saturação) & 5,89 \\
Extrato de saturação & \\
$\quad \mathrm{CE}^{(1)}\left(\mathrm{dS} \mathrm{m}^{-1}\right)$ & 0,71 \\
$\mathrm{RAS}^{(2)}\left(\mathrm{mmol} \mathrm{L}^{-1}\right)^{1 / 2}$ & 0,62 \\
Complexo sortivo $\left(\mathrm{cmol}_{\mathrm{c}} \mathrm{kg}^{-1}\right)$ & \\
$\mathrm{H}+\mathrm{Al}$ & 2,94 \\
$\mathrm{Ca}$ & 2,28 \\
$\mathrm{Mg}$ & 1,51 \\
$\mathrm{Na}$ & 0,04 \\
$\mathrm{~K}$ & 0,04 \\
$\mathrm{CTC}$ & 6,81 \\
$\mathrm{PST}$ & $(3)$ \\
\hline
\end{tabular}

${ }^{(1)}$ Condutividade elétrica do extrato de saturação. ${ }^{(2)}$ Razão de adsorção de sódio. ${ }^{(3)}$ Capacidade de troca catiônica. ${ }^{(4)}$ Porcentagem de sódio trocável. 
mente preparada em laboratório, com a seguinte composição básica: $\mathrm{NaCl}: 20,0 \mathrm{mmol}_{\mathrm{c}} \mathrm{L}^{-1} ; \mathrm{CaCl}_{2} 2 \mathrm{H}_{2} \mathrm{O}$ $8,0 \mathrm{mmol}_{\mathrm{c}} \mathrm{L}^{-1} ; \mathrm{MgCl}_{2} 6 \mathrm{H}_{2} \mathrm{O}: 2,0 \mathrm{mmol}_{\mathrm{c}} \mathrm{L}^{-1} ; \mathrm{NaHCO}_{3}$ : $6,0 \mathrm{mmol}_{\mathrm{c}} \mathrm{L}^{-1}, \mathrm{KHCO}_{3}: 2,0 \mathrm{mmol}_{\mathrm{c}} \mathrm{L}^{-1}, \mathrm{MgSO}_{4} 7 \mathrm{H}_{2} \mathrm{O}$ : 2,0 $\mathrm{mmol}_{\mathrm{c}} \mathrm{L}^{-1}$. Na Tabela 2 constam os dados da análise química dos tratamentos utilizados nas irrigações, realizada segundo Embrapa (1997b).

Foram utilizados bulbilhos da cultivar Cabaceiras, a mais plantada na região de Cabaceiras, $\mathrm{PB}$, selecionados por peso de aproximadamente $1,5 \mathrm{~g}$. Antes do plantio, os bulbilhos foram tratados com fungicidas à base de PCNB e Benomyl, nas doses de 5 e $2 \mathrm{~g} \mathrm{~kg}^{-1}$, respectivamente, para prevenção de doenças.

Foram plantados 15 bulbilhos por vaso, a uma profundidade de 3,0 cm, com o ápice para cima (Brewster, 1994). Após emergência, fez-se o desbaste, deixando-se 10 plantas por vaso.

Aos 35 e 60 dias após plantio (DAP), foram aplicados, respectivamente, 2,4 e $1,8 \mathrm{~g}$ de $\mathrm{N}$ (uréia), em cobertura, com base em Magalhães (1986). Como fonte de P usou-se o ácido fosfórico, na concentração de $10 \mathrm{~mL} \mathrm{~L}^{-1}$ de água, corrigindo-se o $\mathrm{pH}$ para 6,0 com $\mathrm{NH}_{4} \mathrm{OH}$; dessa solução de $\mathrm{P}$ foram aplicados $50 \mathrm{~mL}$ por vaso, aos 20 e $50 \mathrm{DAP}$. O K foi aplicado aos 40 DAP, na dose de 1,2 g de cloreto de potássio por vaso. Foram realizadas três pulverizações foliares com solução a $0,25 \%$ de ácido bórico aos 20,30 e 60 DAP.

No início do ensaio fez-se uma irrigação de acordo com os tratamentos, deixando-se o solo com a umidade no nível da capacidade de campo. Após o plantio, as irrigações basearam-se na pesagem dos vasos, repondo-se a água sempre que havia depleção de $20 \%$ da água disponível A aplicação de água foi suspensa 15 dias antes da colheita.

As plantas foram amostradas em quatro épocas: 30 , 60, 90 e 120 DAP. Nas três primeiras amostragens foram coletadas duas plantas e, na última, quatro. As variáveis estudadas foram: número de folhas verdes, altura, diâmetro do pseudocaule e do bulbo, número de bulbilhos e peso da matéria seca dos bulbos. Na última amostragem foi ob- tido o peso da matéria seca de raízes. A razão bulbar foi obtida dividindo-se o diâmetro do pseudocaule pelo diâmetro do bulbo (Silva \& Alvarenga, 1984; Brewster, 1994). $\mathrm{Na}$ matéria seca das folhas analisaram-se os teores de $\mathrm{K}^{+}$, $\mathrm{Ca}^{2+}, \mathrm{Mg}^{2+}, \mathrm{Na}^{+} \mathrm{e} \mathrm{Cl}^{-}$, conforme Fageria et al. (1991). Ao final do experimento foi feita a análise de amostras do solo de cada tratamento de acordo com Embrapa (1997b).

As análises estatísticas foram realizadas conforme Ferreira (1996), sendo as variáveis número de folhas e número de bulbilhos transformadas em $(\mathrm{x}+1)^{0,5}$; as médias relativas ao modo de aplicação de água foram comparadas pelo teste de Tukey, enquanto as relacionadas a níveis de salinidade, por ser um fator quantitativo, foram analisadas por regressão polinomial.

\section{Resultados e Discussão}

Até 30 DAP as plantas não foram afetadas pelos fatores testados (Tabela 3 ). Mesmo em níveis altos de condutividade elétrica (CEa), todos os bulbilhos plantados brotaram, sem ocorrer diferenças entre os tratamentos nas variáveis analisadas. Não foi verificada significância para a interação NS x MA em qualquer das épocas de amostragem de plantas, indicando que o efeito da salinidade não foi influenciado pelo modo de aplicação de água.

Até 90 DAP não houve efeito significativo do modo de irrigar as plantas, embora já houvesse, na maioria das variáveis, tendência de diminuição dos respectivos valores no tratamento $\mathrm{MA}_{2}$ (Tabela 4). Entretanto, na amostragem realizada no final do ciclo (120 dias), houve redução significativa na altura média das plantas, na fitomassa seca da parte aérea, no diâmetro do pseudocaule e do bulbo, e no número de bulbilhos, com aplicação de água na parte aérea. A redução na fitomassa da parte aérea foi decorrente da menor altura e do menor diâmetro das plantas,

Tabela 2. Análise química das águas utilizadas nos diversos tratamentos de níveis de salinidade (NS).

\begin{tabular}{lcccccccccc}
\hline Tratamento $^{(1)}$ & $\mathrm{pH}$ & $\begin{array}{c}\mathrm{CE} \\
\left.(\mathrm{dS} \mathrm{m})^{-1}\right)\end{array}$ & $\mathrm{Ca}^{2+}$ & $\mathrm{Mg}^{2+}$ & $\mathrm{Na}^{+}$ & $\begin{array}{c}\mathrm{K}^{+} \\
\left(\mathrm{mmol}_{\mathrm{c}} \mathrm{L}^{-1}\right)\end{array}$ & $\mathrm{Cl}^{-}$ & $\mathrm{HCO}_{3}^{-}$ & $\mathrm{CO}_{3}^{2-}$ & $\begin{array}{c}\mathrm{RAS}^{-} \\
\left(\mathrm{mmol} \mathrm{L}^{-1}\right)^{1 / 2}\end{array}$ \\
\hline $\mathrm{NS}_{1}$ & 7,31 & 0,61 & 1,38 & 1,27 & 3,37 & 0,10 & 4,46 & 1,08 & 0,24 & 2,50 \\
$\mathrm{NS}_{2}$ & 7,84 & 1,19 & 2,84 & 2,60 & 6,64 & 0,19 & 8,98 & 2,15 & 0,38 & 4,14 \\
$\mathrm{NS}_{3}$ & 8,11 & 1,80 & 4,27 & 3,07 & 10,94 & 0,49 & 14,00 & 3,22 & 0,64 & 6,48 \\
$\mathrm{NS}_{4}$ & 8,13 & 2,40 & 5,28 & 3,58 & 16,24 & 0,82 & 19,36 & 4,71 & 0,75 & 9,37 \\
$\mathrm{NS}_{5}$ & 8,17 & 3,04 & 6,22 & 4,09 & 21,34 & 1,16 & 23,74 & 5,68 & 0,93 & 11,80 \\
\hline
\end{tabular}

${ }^{(1)} \mathrm{NS}_{1}: 0,6 \mathrm{dS} \mathrm{m}^{-1} ; \mathrm{NS}_{2}: 1,2 \mathrm{dS} \mathrm{m}^{-1} ; \mathrm{NS}_{3}: 1,8 \mathrm{dS} \mathrm{m}^{-1} ; \mathrm{NS}_{4}: 2,4 \mathrm{dS} \mathrm{m}^{-1} ; \mathrm{NS}_{5}: 3,0 \mathrm{dS} \mathrm{m}^{-1}$. 


\begin{tabular}{|c|c|c|c|c|c|c|c|c|c|c|}
\hline \multirow[t]{2}{*}{ Fonte de variação ${ }^{(1)}$} & \multirow[t]{2}{*}{ GL } & \multicolumn{9}{|c|}{ Quadrados médios } \\
\hline & & $\begin{array}{c}\mathrm{N}^{0} \text { de folhas } \\
\text { verdes }^{(2)}\end{array}$ & $\begin{array}{c}\text { Altura das } \\
\text { plantas }(\mathrm{cm})\end{array}$ & $\begin{array}{c}\text { Diâmetro do } \\
\text { pseudocaule (mm) }\end{array}$ & $\begin{array}{l}\text { Diâmetro do } \\
\text { bulbo }(\mathrm{mm})\end{array}$ & $\begin{array}{l}\text { Razão } \\
\text { bulbar }\end{array}$ & $\begin{array}{c}\text { Número de } \\
\text { bulbilhos }\end{array}$ & $\begin{array}{l}\text { Fitomassa da } \\
\text { parte aérea }(\mathrm{g})\end{array}$ & $\begin{array}{l}\text { Fitomassa do } \\
\text { bulbo (g) }\end{array}$ & $\begin{array}{c}\text { Fitomassa } \\
\text { das raízes }(\mathrm{g})\end{array}$ \\
\hline & & \multicolumn{9}{|c|}{ Época 1 (30 DAP) } \\
\hline MA & 1 & 0,0032 & 0,0250 & 0,2250 & - & - & - & 0,0001 & - & - \\
\hline NS & 4 & 0,0009 & 11,1500 & 0,0219 & - & - & - & 0,0009 & - & - \\
\hline MA $x$ NS & 4 & 0,0035 & 11,4391 & 0,1469 & - & - & - & 0,0010 & - & - \\
\hline Blocos & 3 & 0,0184 & 15,1125 & 0,2917 & - & - & - & $0,0022 *$ & - & - \\
\hline Resíduo & 27 & 0,0067 & 10,7410 & 0,1296 & - & - & - & 0,0007 & - & - \\
\hline \multirow[t]{2}{*}{$\mathrm{CV}(\%)$} & & 3,64 & 9,17 & 10,83 & & & & 15,16 & & \\
\hline & & \multicolumn{9}{|c|}{ Época 2 (60 DAP) } \\
\hline MA & 1 & 0,0046 & 7,1402 & 0,0003 & 0,3516 & 0,001 & - & 0,0017 & 0,0001 & - \\
\hline NS & (4) & $0,0141^{*}$ & $48,2966^{* *}$ & $0,3043 *$ & 0,2591 & $0,003 *$ & - & $0,0211^{*}$ & $0,0005^{*}$ & - \\
\hline Regressão linear & 1 & $0,0685^{* *}$ & $165,0662 *$ & $1,0762 *$ & 0,3005 & 0,002 & - & 0,0088 & 0,0003 & - \\
\hline Regressão quadrática & 1 & $0,0182 *$ & 10,8003 & 0,0870 & 0,2206 & 0,001 & - & 0,0012 & 0,0001 & - \\
\hline Desvios de regressão & 2 & 0,0063 & 1,6084 & 0,0002 & 0,0060 & 0,000 & - & 0,0005 & 0,0000 & - \\
\hline MA $x$ NS & 4 & 0,0090 & 1,3418 & 0,0773 & 0,3881 & 0,001 & - & 0,0102 & 0,0001 & - \\
\hline Blocos & 3 & $0,0350 * *$ & $16,2412 * *$ & $0,1592 *$ & 0,2861 & 0,000 & - & 0,0084 & 0,0002 & - \\
\hline Resíduo & 27 & 0,0040 & 5,3058 & 0,0531 & 0,1038 & 0,001 & - & 0,0040 & 0,0001 & - \\
\hline \multirow[t]{2}{*}{$\mathrm{CV}(\%)$} & & 2,63 & 5,64 & 5,70 & 4,29 & 4,44 & - & 13,29 & 9,84 & - \\
\hline & & \multicolumn{9}{|c|}{ Época 3 (90 DAP) } \\
\hline MA & 1 & 0,0123 & 8,9303 & 0,1051 & 0,6126 & 0,002 & - & 0,0342 & 0,0031 & - \\
\hline NS & (4) & $0,1031 * *$ & $99,0448^{* *}$ & $1,5596 * *$ & $3,6753^{* *}$ & $0,003 *$ & - & $0,4085^{* *}$ & $0,0094 *$ & - \\
\hline Regressão linear & 1 & $0,4025 * *$ & $192,0805^{*}$ & $6,2203^{* *}$ & $4,0570 * *$ & 0,002 & - & $1,6050^{* * *}$ & $0,0496 * *$ & - \\
\hline Regressão quadrática & 1 & 0,0130 & 10,1550 & 0,2980 & 0,6005 & 0,001 & - & $0,8802 * *$ & $0,0124 *$ & - \\
\hline Desvios de regressão & 2 & 0,0018 & 0,8051 & 0,0605 & 0,0882 & 0,000 & - & 0,0050 & 0,0005 & - \\
\hline MA x NS & 4 & 0,0165 & 8,4360 & 0,2060 & 0,5048 & 0,001 & - & 0,0495 & 0,0038 & - \\
\hline Blocos & 3 & $0,0850 * *$ & 14,0905 & 0,4507 & 0,0787 & 0,002 & - & 0,1250 & 0,0021 & - \\
\hline Resíduo & 27 & 0,0122 & 7,4180 & 0,1766 & 0,5848 & 0,001 & - & 0,0326 & 0,0033 & - \\
\hline \multirow[t]{2}{*}{$\mathrm{CV}(\%)$} & & 4,43 & 5,84 & 9,14 & 6,46 & 8,30 & - & 19,05 & 14,85 & - \\
\hline & & \multicolumn{9}{|c|}{ Época 4 (120 DAP) } \\
\hline MA & 1 & - & $57,5520 * *$ & $0,6917 * *$ & $4,9280^{*}$ & 0,0004 & $0,3641^{* *}$ & $0,2117 * *$ & $0,3820 * *$ & 0,0013 \\
\hline NS & (4) & - & $108,4181 * *$ & $1,2408 * *$ & $21,7371 * *$ & 0,0001 & $0,5250 * *$ & $0,2024 * *$ & $0,4287 * *$ & $0,0120^{*}$ \\
\hline Regressão linear & 1 & - & $415,0061^{*}$ & $4,6754 * *$ & $80,4005^{* *}$ & 0,0001 & $2,0690 * *$ & $0,7488 * *$ & $1,6103 * *$ & $0,0449 * *$ \\
\hline Regressão quadrática & 1 & - & 14,8556 & 0,2074 & $5,2550^{*}$ & 0,0000 & 0,0204 & $0,0417 *$ & 0,0897 & 0,0012 \\
\hline Desvios de regressão & 2 & - & 1,9054 & 0,0402 & 0,6465 & 0,0000 & 0,0053 & 0,0095 & 0,0074 & 0,0009 \\
\hline MA $x$ NS & 4 & - & 2,5032 & 0,0418 & 0,6634 & 0,0004 & 0,0267 & 0,0016 & 0,0315 & 0,0019 \\
\hline Blocos & 3 & - & 2,8514 & 0,0381 & 1,0336 & 0,0003 & 0,0496 & 0,0032 & 0,0256 & 0,0029 \\
\hline Resíduo & 27 & - & 4,4482 & 0,0631 & 0,9152 & 0,0003 & 0,0485 & 0,0068 & 0,0375 & 0,0031 \\
\hline $\mathrm{CV}(\%)$ & & & 5,28 & 7,72 & 6,39 & 7,21 & 8,34 & 18,84 & 15,32 & 14,54 \\
\hline
\end{tabular}

(1)MA: modo de aplicação da água de irrigação; NS: níveis de salinidade; DAP: dias após plantio. ${ }^{(2)}$ Dados transformados em $(\mathrm{x}+1)^{0,5}$ * e ** Significativo a $5 \%$ e a $1 \%$ de probabilidade, respectivamente, pelo teste $\mathrm{F}$. 
sendo tais efeitos da salinidade já registrados por Maas (1986); a produção de fitomassa da parte aérea foi reduzida em 19,72\% com aplicação de água na folhagem, enquanto a diminuição na fitomassa de bulbos foi de $12,8 \%$. Os bulbos produzidos tinham, também, menor diâmetro e menor número de bulbilhos.

Esse efeito prejudicial de $\mathrm{MA}_{2}$ deveu-se à acumulação de íons nas folhas, principalmente de $\mathrm{Na}^{+} \mathrm{e}$ $\mathrm{Cl}^{-}$, predominantes na água de irrigação, conforme análise foliar (Tabela 5). No caso de molhamento das

Tabela 4. Valores médios das diversas variáveis estudadas, em razão do modo de aplicação da água de irrigação(1)

\begin{tabular}{|c|c|c|c|}
\hline \multirow{2}{*}{$\begin{array}{l}\text { Época de } \\
\text { amostragem } \\
\text { (dias após } \\
\text { plantio) }\end{array}$} & \multicolumn{2}{|c|}{$\begin{array}{l}\text { Modo de aplicação da } \\
\text { água de irrigação }\end{array}$} & \multirow[t]{2}{*}{$\begin{array}{c}\text { Redução relativa } \\
\left\{\left[\left(\mathrm{MA}_{2}\right) \times\left(\mathrm{MA}_{1}\right)^{-1}\right] \times 100\right\}-100\end{array}$} \\
\hline & $\mathrm{MA}_{1}$ & $\mathrm{MA}_{2}$ & \\
\hline & \multicolumn{3}{|c|}{ Número de folhas verdes } \\
\hline 30 & $2,27 \mathrm{a}$ & $2,25 \mathrm{a}$ & $-0,88 \%$ \\
\hline 60 & $2,40 \mathrm{a}$ & $2,42 \mathrm{a}$ & $0,83 \%$ \\
\hline 90 & $2,50 \mathrm{a}$ & $2,47 \mathrm{a}$ & $-1,20 \%$ \\
\hline \multirow[t]{2}{*}{$120^{(3)}$} & - & - & - \\
\hline & \multicolumn{3}{|c|}{ Altura das plantas $(\mathrm{cm})$} \\
\hline 30 & $35,75 \mathrm{a}$ & $35,70 \mathrm{a}$ & $-0,14 \%$ \\
\hline 60 & $41,43 a$ & $41,27 \mathrm{a}$ & $-0,39 \%$ \\
\hline 90 & $47,11 \mathrm{a}$ & $46,16 a$ & $-2,02 \%$ \\
\hline \multirow[t]{2}{*}{120} & $47,22 \mathrm{a}$ & $44,82 \mathrm{~b}$ & $-5,08 \% * *$ \\
\hline & \multicolumn{3}{|c|}{ Diâmetro do pseudocaule (mm) } \\
\hline 30 & $3,25 \mathrm{a}$ & $3,40 \mathrm{a}$ & $4,62 \%$ \\
\hline 60 & $4,05 \mathrm{a}$ & $4,04 \mathrm{a}$ & $-0,25 \%$ \\
\hline 90 & $4,65 \mathrm{a}$ & $4,54 \mathrm{a}$ & $-2,37 \%$ \\
\hline \multirow[t]{2}{*}{120} & $3,87 \mathrm{a}$ & $3,60 \mathrm{~b}$ & $-6,98 \% * *$ \\
\hline & \multicolumn{3}{|c|}{ Diâmetro do bulbo (mm) } \\
\hline 30 & $7,42 \mathrm{a}$ & $7,60 \mathrm{a}$ & $2,42 \%$ \\
\hline 60 & $11,96 \mathrm{a}$ & $11,72 \mathrm{a}$ & $2,05 \%$ \\
\hline \multirow[t]{2}{*}{90} & $15,32 \mathrm{a}$ & $14,62 \mathrm{~b}$ & $-4,57 \%$ * \\
\hline & \multicolumn{3}{|c|}{ Razão bulbar } \\
\hline 30 & $0,44 \mathrm{a}$ & $0,45 \mathrm{a}$ & $2,27 \%$ \\
\hline 60 & $0,34 \mathrm{a}$ & $0,34 \mathrm{a}$ & $0,00 \%$ \\
\hline \multirow[t]{2}{*}{90} & $0,30 \mathrm{a}$ & $0,31 \mathrm{a}$ & $3,33 \%$ \\
\hline & \multicolumn{3}{|c|}{ Número de bulbilhos } \\
\hline \multirow[t]{2}{*}{120} & $2,74 \mathrm{a}$ & $2,54 \mathrm{~b}$ & $-7,30 \% * *$ \\
\hline & \multicolumn{3}{|c|}{ Fitomassa seca da parte aérea (g) } \\
\hline 30 & $0,17 \mathrm{a}$ & $0,17 \mathrm{a}$ & $0,00 \%$ \\
\hline 60 & $0,48 \mathrm{a}$ & $0,47 \mathrm{a}$ & $-2,08 \%$ \\
\hline 90 & $0,98 \mathrm{a}$ & $0,92 \mathrm{a}$ & $-6,12 \%$ \\
\hline 120 & $0,71 \mathrm{a}$ & $0,57 \mathrm{~b}$ & $-19,72 \% * *$ \\
\hline & \multicolumn{3}{|c|}{ Fitomassa seca do bulbo (g) } \\
\hline 60 & $0,11 \mathrm{a}$ & $0,10 \mathrm{a}$ & $-9,09 \%$ \\
\hline 90 & $0,39 \mathrm{a}$ & $0,38 \mathrm{a}$ & $-2,56 \%$ \\
\hline \multirow[t]{2}{*}{120} & $0,78 \mathrm{a}$ & $0,68 \mathrm{~b}$ & $-12,82 \% * *$ \\
\hline & \multicolumn{3}{|c|}{ Fitomassa seca das raízes (g) } \\
\hline 120 & $0,24 \mathrm{a}$ & $0,22 \mathrm{a}$ & $-8,33 \%$ \\
\hline
\end{tabular}

(1)Médias seguidas de mesma letra na horizontal não diferem, entre si, a 5\% de probabilidade, pelo teste de Tukey. (2) $\mathrm{MA}_{1}$ : aplicação de água no solo $\mathrm{MA}_{2}$ : aplicação de água molhando as folhas. ${ }^{(3)}$ Aos 120 DAP, época da colheita, não havia folhas verdes. * e **Significativo a $5 \%$ e a $1 \%$ de probabilidade pelo teste de Tukey, respectivamente. folhas, o teor de $\mathrm{Na}$ aumentou em $65,0 \%$ enquanto o $\mathrm{Cl}^{-}$, em apenas 8,7\%. Magalhães (1986) e François (1994) citam ser o alho muito sensível ao íon cloreto.

A folhagem das plantas, ao ser molhada com água salina, além dos efeitos resultantes da própria salinidade, está sujeita a danos adicionais, causados pelo acúmulo nas células dos tecidos foliares de íons tóxicos, como o $\mathrm{Na}^{+}$e o $\mathrm{Cl}^{-}$, absorvidos diretamente pelas folhas (Grattan et al., 1981; Maas, 1986; Katerji et al., 1996).

Segundo Maas (1986), a suscetibilidade ao dano foliar, resultante de irrigação por aspersão com águas salinas, varia consideravelmente entre as espécies, dependendo mais da taxa de absorção foliar e das características da folha do que, propriamente, da tolerância da planta à salinidade do solo.

$\mathrm{O}$ fato de não ter havido influência do modo de aplicação da água até os 90 dias pode ser devido à arquitetura foliar do alho, com posicionamento mais ereto das folhas, nas primeiras fases do ciclo, aliado ao seu tipo lanceolado, com diferentes graus de cerosidade (Brewster, 1994). Na fase final do ciclo, as folhas tendem a se inclinar, favorecendo a um tempo maior de retenção de água na superfície da folha, o que, segundo Grattan et al. (1981), resulta em maiores danos às plantas.

Segundo Fageria et al. (1991), a parte aérea da planta é mais afetada pela salinidade do que as raízes. Sendo o bulbo de alho uma estrutura especial, botanicamente considerado como caule subterrâneo modificado (Brewster, 1994), observa-se que, mesmo sem diferença estatística, a fitomassa do bulbo foi mais afetada (redução de $12 \%$ ) pela acumulação de sais nas folhas do que as raízes, cuja fitomassa teve redução de apenas $8,3 \%$.

Com relação aos níveis de salinidade, surgiram efeitos só a partir de 60 DAP, sendo afetados linearmente a altura de plantas e o diâmetro do pseudocaule (Tabelas 3 e 6). François (1994) verificou também ser a altura das plantas a variável vegetativa mais afetada pela salinidade. Sobre o número de folhas o efeito foi quadrático, sendo favorecido pelos sais até o nível de condutividade elétrica da água de irrigação de $1,1 \mathrm{dS} \mathrm{m}^{-1}$ (Tabela 6). Entretanto, com a continuação da aplicação de água salina, houve diminuição da emissão de folhas, com efeito linear da concentração de sais, aos 90 DAP. Entre outros autores, Lazof \& 
Bernstein (1999) citam que a emissão de folhas das plantas é muito afetada pela salinidade.

Quanto à razão bulbar, uma das características de desenvolvimento do bulbo (Silva \& Alvarenga, 1984; Brewster, 1994), não houve efeito dos tratamentos, pelo fato de ter ocorrido redução, tanto no diâmetro do pseudocaule quanto do bulbo, à medida que aumentou a concentração salina (Tabela 6).

As variáveis relacionadas a bulbos e bulbilhos não foram afetadas pelos tratamentos de salinidade até os 60 DAP (Tabelas 3 e 6), coincidindo com o início de formação do bulbo; as maiores influências surgiram a partir de 90 DAP.

A fitomassa da parte aérea começou a ser mais afetada a partir de 90 dias (Tabela 6). Nos últimos 30 dias do ciclo (entre 90 e 120 dias), reduziu-se a fitomassa da parte aérea e aumentou a do bulbo, provavelmente devido à translocação de fotoassimilados para a estrutura bulbar, em fase final de formação. Este fato foi também observado por Silva et al. (1970), estudando a absorção de nutrientes em todo o ciclo da cultura de alho. No nível mais baixo de salinidade $\left(0,6 \mathrm{dS} \mathrm{m}^{-1}\right)$, ocorreu redução da fitomassa da parte aérea em $24,6 \%$, chegando a $26,2 \%$ na concentração mais alta de sais $\left(3,0 \mathrm{dS} \mathrm{m}^{-1}\right)$. Enquanto isso, nesse mesmo período, o incremento de fitomassa do bulbo foi de $175 \%$ no tratamento de $0,6 \mathrm{dS} \mathrm{m}^{-1}$, e de apenas $50 \%$ com a utilização de água com CEa de $3,0 \mathrm{dS} \mathrm{m}^{-1}$ (Tabela 6). Com o aumento da salinidade, certamente diminuiu o potencial osmótico da solução do solo, dificultando a absorção de água pelas raízes, aliado ao fato de terem sido acumulados íons $\mathrm{Na}^{+}$e $\mathrm{Cl}^{-}$nas folhas, nos níveis mais altos de salinidade (Tabela 5), afetando os processos fisiológicos da planta
(Noble \& Rogers, 1992; Lazof \& Bernstein, 1999). Em relação ao nível mais baixo de $\mathrm{CEa}$, as plantas irrigadas com água de $3,0 \mathrm{dS} \mathrm{m}^{-1}$ acumularam $223 \%$ a mais de $\mathrm{Na}^{+}$e $91 \%$ a mais de cloreto.

$\mathrm{O}$ aumento do teor de $\mathrm{Na}^{+}$no tecido foliar não influenciou a concentração de $\mathrm{K}^{+}$nas folhas do alho. Esses resultados são semelhantes aos de Singh \& Abrol (1985). Todavia, o teor de $\mathrm{Ca}^{2+}$ foi bastante elevado em relação ao obtido por Silva et al. (1970), que conduziram ensaio em solução nutritiva. Vale lembrar que o Ca apresenta seletividade maior que o $\mathrm{Na}^{+}$, fato também já registrado na literatura (Kinraide, 1999).

Vários autores se referem ao efeito osmótico da salinidade, bem como ao efeito de íons específicos quando plantas são cultivadas em condições de estresse salino (Jacoby, 1993; Shalhevet et al., 1995; Rao, 1999). Magalhães (1986) e François (1994) citam ser o alho muito sensível ao íon cloreto. Singh \& Abrol (1985) verificaram que o alho é sensível também a níveis crescentes de $\mathrm{Na}^{+}$, através da redução do diâmetro e do peso dos bulbos.

Quanto ao diâmetro e à fitomassa de bulbos, os efeitos da salinidade foram mais prejudiciais nos últimos 30 dias do ciclo. Aos 90 DAP, o acúmulo de matéria seca no bulbo foi favorecido pela salinidade da água de irrigação, até a CEa de 1,3 dS m² , baixando nos tratamentos com maior concentração de sais; em relação ao tratamento de menor concentração $\left(0,6 \mathrm{dS} \mathrm{m}^{-1}\right)$, o nível crítico foi de $1,66 \mathrm{dS} \mathrm{m}^{-1}$, a partir do qual passou a ser afetada a acumulação de fitomassa no bulbo (Tabela 6). Enquanto a diferença no diâmetro, aos $90 \mathrm{DAP}$, entre os dois níveis extremos de $\mathrm{CEa}\left(0,6\right.$ e 3,0 dS m $\left.\mathrm{d}^{-1}\right)$, foi de apenas $13,0 \%$,

Tabela 5. Teores de cálcio, magnésio, sódio, potássio e cloreto nas folhas de alho, em razão do modo de aplicação (MA) e dos níveis de salinidade (NS) da água de irrigação, aos 120 dias após o plantio.

\begin{tabular}{|c|c|c|c|c|c|c|c|}
\hline \multirow[t]{2}{*}{ Elemento } & \multicolumn{2}{|c|}{ Modo de aplicação ${ }^{(1)}$} & \multicolumn{5}{|c|}{ Nível de salinidade da água ${ }^{(2)}$} \\
\hline & $\mathrm{MA}_{1}$ & $\mathrm{MA}_{2}$ & $\mathrm{NS}_{1}$ & $\mathrm{NS}_{2}$ & $\mathrm{NS}_{3}$ & $\mathrm{NS}_{4}$ & $\mathrm{NS}_{5}$ \\
\hline & \multicolumn{7}{|c|}{ 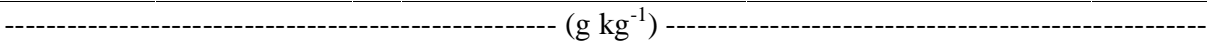 } \\
\hline Cálcio & 36,0 & 36,6 & 38,3 & 36,3 & 35,3 & 36,0 & 36,0 \\
\hline Magnésio & 3,2 & 3,0 & 3,5 & 3,1 & 2,8 & 2,9 & 2,7 \\
\hline Sódio & 2,0 & 3,3 & 1,3 & 2,1 & 2,2 & 3,7 & 4,2 \\
\hline Potássio & 44,4 & 46,4 & 46,8 & 48,2 & 44,3 & 45,3 & 42,4 \\
\hline Cloreto & 58,6 & 63,7 & 39,4 & 53,8 & 62,1 & 74,9 & 75,5 \\
\hline
\end{tabular}

${ }^{(1)} \mathrm{MA}_{1}$ : aplicação de água no solo; $\mathrm{MA}_{2}$ : aplicação de água molhando as folhas. (2) $\mathrm{NS}_{1}: 0,6 \mathrm{dS} \mathrm{m}^{-1} ; \mathrm{NS}_{2}: 1,2 \mathrm{dS} \mathrm{m}^{-1} ; \mathrm{NS}_{3}: 1,8 \mathrm{dS} \mathrm{m}^{-1} ; \mathrm{NS}_{4}: 2,4 \mathrm{dS} \mathrm{m}^{-1}$; $\mathrm{NS}_{5}: 3,0 \mathrm{dS} \mathrm{m}$. $^{-1}$ 
no final do ciclo essa diferença aumentou para $23,2 \%$; esses valores para fitomassa de bulbos são respectivamente de $16,5 \%$ e $54,4 \%$ (Tabela 6). Mangal et al. (1990) e François (1994) verificaram também maior efeito da salinidade sobre a redução da fitomassa de bulbos entre as variáveis de produção.

Foi intensa a acumulação de fitomassa seca nos bulbos (Tabela 6), nos últimos 30 dias do ciclo, prin- cipalmente nos níveis mais baixos de $\mathrm{CEa}$; no tratamento $\mathrm{NS}_{1}\left(0,6 \mathrm{dS} \mathrm{m}^{-1}\right)$ houve aumento de $175 \%$, baixando para $93,2 \%$, em $1,2 \mathrm{dS} \mathrm{m}^{-1}$, e para $71,4 \%$, em $1,8 \mathrm{dS} \mathrm{m}^{-1}$, enquanto no nível mais alto de $\mathrm{CEa}$ $\left(3,0 \mathrm{dS} \mathrm{m}^{-1}\right)$ a matéria seca do bulbo aumentou apenas $50 \%$. Calculando-se o incremento diário de fitomassa seca do bulbo, entre 90 e 120 DAP, verifica-se que na concentração de $0,6 \mathrm{dS} \mathrm{m} \mathrm{m}^{-1}$ ocorreu acu-

Tabela 6. Médias e equações matemáticas obtidas através de regressão polinomial para as variáveis estudadas de alho, nas diversas épocas de desenvolvimento das plantas.

\begin{tabular}{|c|c|c|c|c|c|c|c|}
\hline \multirow{2}{*}{$\begin{array}{c}\text { Dias após } \\
\text { plantio }\end{array}$} & \multicolumn{5}{|c|}{ Nível de salinidade da água de irrigação $\left(\mathrm{dS} \mathrm{m}^{-1}\right)$} & \multirow[t]{2}{*}{ Equação de regressão } & \multirow[t]{2}{*}{$\mathrm{R}^{2}$} \\
\hline & $\mathrm{NS}_{1}=0,6$ & $\mathrm{NS}_{2}=1,2$ & $\mathrm{NS}_{3}=1,8$ & $\mathrm{NS}_{4}=2,4$ & $\mathrm{NS}_{5}=3,0$ & & \\
\hline & \multicolumn{7}{|c|}{ Número de folhas ${ }^{(1)}$} \\
\hline 30 & 2,2742 & 2,2484 & 2,2627 & 2,2634 & 2,2497 & ns & \\
\hline 60 & 2,4354 & 2,4735 & 2,4405 & 2,3837 & 2,3713 & $y=-0,0248 x^{2}+0,0528 x+2,4239$ & 0,81 \\
\hline \multirow[t]{2}{*}{90} & 2,6065 & 2,5240 & 2,5606 & 2,4174 & 2,3270 & $y=-0,1109 x+2,6868$ & 0,86 \\
\hline & \multicolumn{7}{|c|}{ Altura de plantas $(\mathrm{cm})$} \\
\hline 30 & 36,5625 & 36,5938 & 33,8438 & 36,3438 & 35,2813 & ns & \\
\hline 60 & 43,6188 & 41,5313 & 42,5313 & 38,4000 & 38,1688 & $y=-2,3383 x+45,059$ & 0,82 \\
\hline 90 & 49,9625 & 50,4313 & 46,7000 & 44,5125 & 41,8688 & $y=-3,6834 x+53,327$ & 0,93 \\
\hline \multirow[t]{2}{*}{120} & 51,3113 & 47,3775 & 45,7613 & 42,9238 & 42,3488 & $y=-3,7298 x+52,658$ & 0,95 \\
\hline & \multicolumn{7}{|c|}{ Diâmetro do pseudocaule (mm) } \\
\hline 30 & 3,3750 & 3,3125 & 3,2500 & 3,3750 & 3,3125 & ns & \\
\hline 60 & 4,2625 & 4,0563 & 4,2001 & 3,9125 & 3,7938 & $y=-0,1802 x+4,3694$ & 0,77 \\
\hline 90 & 5,0500 & 4,8875 & 4,7875 & 4,2125 & 4,0438 & $y=-0,45 x+5,406$ & 0,92 \\
\hline \multirow[t]{2}{*}{120} & 4,3125 & 3,8988 & 3,7213 & 3,3938 & 3,3563 & $y=-0,4017 x+4,459$ & 0,94 \\
\hline & \multicolumn{7}{|c|}{ Diâmetro do bulbo $(\mathrm{mm})$} \\
\hline 60 & 7,6125 & 7,4125 & 7,7625 & 7,3000 & 7,4688 & ns & \\
\hline 90 & 12,6625 & 12,3875 & 11,7000 & 11,4438 & 11,0123 & $y=-0,7074 x+13,114$ & 0,98 \\
\hline \multirow[t]{2}{*}{120} & 17,4763 & 15,5150 & 14,8163 & 13,6075 & 13,4175 & $y=-0,6017 x^{2}-3,8369 x+19,49$ & 0,99 \\
\hline & \multicolumn{7}{|c|}{ Razão bulbar } \\
\hline 60 & 0,5600 & 0,5475 & 0,5413 & 0,5363 & 0,5075 & ns & \\
\hline 90 & 0,4000 & 0,3950 & 0,4113 & 0,3675 & 0,3688 & ns & \\
\hline \multirow[t]{2}{*}{120} & 0,2475 & 0,2513 & 0,2500 & 0,2500 & 0,2525 & ns & \\
\hline & \multicolumn{7}{|c|}{ Número de bulbilhos ${ }^{(1)}$} \\
\hline \multirow[t]{2}{*}{120} & 3,3800 & 2,4413 & 2,2175 & 1,8538 & 1,7450 & $y=-0,27 x+3,12$ & 0,97 \\
\hline & \multicolumn{7}{|c|}{ Fitomassa da parte aérea $(\mathrm{g})$} \\
\hline 30 & 0,1763 & 0,1750 & 0,1588 & 0,1863 & 0,1638 & ns & \\
\hline 60 & 0,5325 & 0,4663 & 0,5263 & 0,4238 & 0,4313 & ns & \\
\hline 90 & 1,1700 & 1,1650 & 0,9525 & 0,7925 & 0,6588 & $y=-0,0407 x^{2}-0,0861 x+1,2638$ & 0,97 \\
\hline \multirow[t]{2}{*}{120} & 0,8825 & 0,6863 & 0,6350 & 0,5113 & 0,4863 & $y=0,0536 x^{2}-0,3541 x+1,0655$ & 0,98 \\
\hline & \multicolumn{7}{|c|}{ Fitomassa do bulbo $(\mathrm{g})$} \\
\hline 60 & 0,1025 & 0,1023 & 0,1188 & 0,1000 & 0,1025 & ns & \\
\hline 90 & 0,3950 & 0,4200 & 0,4025 & 0,3788 & 0,3300 & $y=-0,0305 x^{2}+0,0813 x+0,3597$ & 0,98 \\
\hline 120 & 1,0863 & 0,8113 & 0,6900 & 0,5750 & 0,4950 & $y=-0,2365 x+1,1572$ & 0,93 \\
\hline & \multicolumn{7}{|c|}{ Fitomassa de raiz $(\mathrm{g})$} \\
\hline 120 & 0,3050 & 0,2745 & 0,2325 & 0,1775 & 0,1725 & $y=-0,0558 x+0,3275$ & 0,94 \\
\hline
\end{tabular}

${ }^{(1)}$ Dados transformados em $(\mathrm{x}+1)^{0,5}$. ${ }^{\text {ns }}$ Não-significativo pelo teste $\mathrm{F}$. 
mulação de $23 \mathrm{mg} \mathrm{dia}^{-1}$, diminuindo para $13 \mathrm{mg} \mathrm{dia}^{-1}$ no tratamento de $1,2 \mathrm{dS} \mathrm{m}^{-1}$, enquanto no nível mais alto de salinidade $\left(3,0 \mathrm{dS} \mathrm{m}^{-1}\right)$ o incremento diário foi de apenas 5,5 $\mathrm{mg} \mathrm{dia}^{-1}$. Segundo alguns autores (Hoorn et al., 1993; Jacoby, 1993; Sultana \& Ikeda, 1999), em estresse salino as plantas gastam parte da energia metabólica para se ajustar às condições adversas do meio, o que explica as diferenças de acumulação de fitomassa obtidas neste trabalho, com o aumento dos níveis de $\mathrm{CEa}$.

Quanto ao sistema radicular, observa-se ter havido efeito linear decrescente da salinidade sobre a fitomassa seca de raízes. Obtendo-se a relação raiz/ parte aérea no final do ciclo, a partir dos dados de fitomassa dos tratamentos extremos $\left(0,6\right.$ e $\left.3,0 \mathrm{dS} \mathrm{m}^{-1}\right)$, verifica-se que, aumentando a salinidade, a parte aérea foi ligeiramente mais afetada que o sistema radicular. Tais resultados corroboram dados encontrados em Ludlow \& Muchow (1990), Fageria et al. (1991) e Shalhevet et al. (1995). Vale ser ressaltado que o bulbo é uma estrutura modificada de caule (Brewster, 1994), não fazendo parte do sistema radicular.

Houve grande aumento na concentração de sais no solo, variando de 7,6 a 23,4 vezes, dependendo da CEa, com correspondente incremento nos valores de PST, o que era esperado, uma vez que no período de ensaio não se permitiu qualquer lavagem ou drenagem (Tabela 7). O fator de concentração de sais no solo (CEes/CEa) diminuiu com o aumento da salinidade da água, provavelmente decorrente da precipitação de parte dos sais em forma de carbonato de cálcio e magnésio ou sulfato de cálcio (Ayers \& Westcot, 1991), e também pelo fato da aplicação de menor volume de água nesses tratamentos em relação ao controle. O aumento da PST no solo foi em proporção ao aumento de $\mathrm{Na}^{+}$na água de irrigação, alcançando valor de $26,2 \%$ no caso de água $\mathrm{NS}_{5}$; o valor de $\mathrm{pH}_{\mathrm{ps}}$ baixou em relação ao seu nível original, com diminuições mais apreciáveis até o nível $\mathrm{NS}_{3}$, em virtude do efeito residual de adubos aplicados.

É interessante observar que enquanto a CEes aumentou pelo fator de 3,08 entre $\mathrm{NS}_{1}$ e $\mathrm{NS}_{5}$, a PST foi 5,02 vezes maior, o que confirma que no nível mais elevado de CEa deve ter havido precipitação de parte de $\mathrm{Ca}$ e $\mathrm{Mg}$, proporcionando um aumento relativo na concentração de $\mathrm{Na}^{+}$solúvel na solução, responsável pela elevação da PST.

Mangal et al. (1990) encontraram o valor de salinidade limiar para alho igual a $1,70 \mathrm{dS} \mathrm{m}^{-1}$ e declínio na produção de $1,68 \%$ a cada unidade de aumento na CEes acima do limiar. Os mesmos autores verificaram, dependendo da cultivar, queda na produção da ordem de $50 \%$, na faixa de CEes entre 5,60 e 7,80 dS m${ }^{-1}$. Tais valores diferem dos obtidos no presente trabalho, pois no final do experimento (120 dias), para CEes variando de 5,40 e 16,63 $\mathrm{dS} \mathrm{m}^{-1}$ (Tabela 7), observou-se uma diminuição na fitomassa seca do bulbo de 54,4\% (Tabela 6); considerando-se os dois níveis mais altos de salinidade estudados, constata-se um decréscimo relativo de $4,8 \%$ por aumento unitário da salinidade do solo (CEes) acima de $5,40 \mathrm{dS} \mathrm{m}^{-1}$. Por sua vez, François (1994), ao estudar dois genótipos de alho, encontrou para um deles o valor de salinidade limiar de $4,2 \mathrm{dS} \mathrm{m}^{-1}$ e decréscimo de $14,3 \%$ por unidade de incremento da salinidade acima desse limiar. As diferenças, entre outros fatores, devem-se às práticas de manejo de água e às diferenças entre genótipos.

Tabela 7. Dados de caracterização química do material de solo, antes e após a realização do experimento, em razão do modo de aplicação da água (MA) e dos níveis de salinidade da água de irrigação (NS).

\begin{tabular}{|c|c|c|c|c|c|c|c|c|}
\hline \multirow[t]{3}{*}{ Característica $^{(1)}$} & \multirow{3}{*}{$\begin{array}{l}\text { Antes do } \\
\text { experimento }\end{array}$} & \multicolumn{7}{|c|}{ Após o experimento } \\
\hline & & \multicolumn{2}{|c|}{ Modo de aplicação ${ }^{(2)}$} & \multicolumn{5}{|c|}{ Nível de salinidade ${ }^{(3)}$} \\
\hline & & $\mathrm{MA}_{1}$ & $\mathrm{MA}_{2}$ & $\mathrm{NS}_{1}$ & $\mathrm{NS}_{2}$ & $\mathrm{NS}_{3}$ & $\mathrm{NS}_{4}$ & $\mathrm{NS}_{5}$ \\
\hline $\mathrm{pH}_{\mathrm{ps}}$ & 5,88 & 5,06 & 4,99 & 4,83 & 4,93 & 4,95 & 5,11 & 5,31 \\
\hline CEes $\left(\mathrm{dS} \mathrm{m}^{-1}\right)$ & 0,71 & 10,97 & 11,94 & 5,40 & 9,14 & 10,99 & 15,13 & 16,63 \\
\hline PST & 0,59 & 15,77 & 17,24 & 5,22 & 12,26 & 15,65 & 20,65 & 26,20 \\
\hline
\end{tabular}

${ }^{(1)} \mathrm{pH}_{\mathrm{ps}}$ : $\mathrm{pH}$ da pasta saturada; CEes: condutividade elétrica do extrato de saturação do solo; PST: porcentagem de sódio trocável. (2) $\mathrm{MA}_{1}$ : aplicação da água no solo; $\mathrm{MA}_{2}$ : aplicação da água molhando a folhagem. ${ }^{(3)} \mathrm{NS}_{1}: 0,6 \mathrm{dS} \mathrm{m}^{-1}, \mathrm{NS}_{2}: 1,2 \mathrm{dS} \mathrm{m}^{-1}, \mathrm{NS}_{3}: 1,8 \mathrm{dS} \mathrm{m}^{-1}, \mathrm{NS}_{4}: 2,4 \mathrm{dS} \mathrm{m}^{-1}, \mathrm{NS}_{5}: 3,0 \mathrm{dS} \mathrm{m}^{-1}$. 


\section{Conclusões}

1. Molhando-se a folhagem das plantas diminui-se o crescimento da parte aérea, a fitomassa do bulbo e o número de bulbilhos, com efeito apenas no final do ciclo.

2. A cultivar Cabaceiras é relativamente tolerante à salinidade na fase de brotação de bulbilhos e crescimento inicial das plantas, até 30 dias após plantio.

3. A fase mais sensível do alho à salinidade é a do período final de formação do bulbo, ou seja, os últimos 30 dias do ciclo.

4. A razão bulbar não é um bom parâmetro para avaliação de tolerância do alho à salinidade.

\section{Referências}

AYERS, R. S.; WESTCOT, D. W. A qualidade da água na agricultura. Tradução de Hans Raj Gheyi, José Francismar de Medeiros, Francisco Ademilton Damasceno Campina Grande: UFPB, 1991. 218 p. (Estudos da FAO, Irrigação e Drenagem, 29).

BREWSTER, J. L. Onions and other vegetables alliums. Wallingford: CAB International, 1994. $236 \mathrm{p}$

EMBRAPA. Centro Nacional de Pesquisa de Hortaliças (Brasília, DF). Cultivo do alho (Allium sativum L.) Brasília: Embrapa-CNPH, 1997a. 24 p. (Instruções Técnicas, 2)

EMBRAPA. Centro Nacional de Pesquisa de Solos (Rio de Janeiro, RJ). Manual de métodos de análise de solo Rio de Janeiro, 1997b. 297 p.

FAGERIA, N. K.; BALIGAR, V. C.; JONES, C. A Growth and mineral nutrition of field crops. New York: M. Dekker, 1991. 476 p.

FERREIRA, P. V. Estatística experimental aplicada à agronomia. 2. ed. Maceió: Ed. da Ufal, 1996. 606 p.

FRANÇOIS, L. E. Yield and quality response of saltstressed garlic. HortScience, Alexandria, v. 29, n. 11, p. 1314-1317, 1994

GRATTAN, S.; MAAS, E. V.; OGATA, G. Foliar uptake and injury from saline aerosol. Journal of Environmental Quality, Madison, v. 10, p. 406-409, 1981

HOORN, J. W. van; KATERJI, N.; HAMDY, A. MASTRORILLI, M. Effect of saline water on soil salinity and on water stress, growth, and yield of wheat and potatoes. Agricultural Water Management, Amsterdam, v. 23, n. 3 , p. $247-265,1993$

JACOBY, B. Mechanisms involved in salt tolerance by plants. In: PESSARAKLI, M. (Ed.). Handbook of plant and crop stress. New York: M. Dekker, 1993. p. 97-124.

KATERJ, N.; HOORN, J. W. van; HAMDY, A. Effect of salinity on water stress, growth and yield of maize and sunflower. Agricultural Water Management, Amsterdam, v. 30, n. 3, p. 237-249, 1996.

KINRAIDE, T. B. Interactions among $\mathrm{Ca}^{2+}, \mathrm{Na}^{+}$and $\mathrm{K}^{+}$in salinity toxicity: quantitative resolution of multiple toxic and ameliorative effects. Journal of Experimental Botany, Oxford, v. 50, n. 3, p. 1495-1505, 1999.

LAZOF, D. B.; BERNSTEIN, N. Effects of salinization on nutrient transport to lettuce leaves: consideration of leaf developmental stage. The New Phytologist, Cambridge, Inglaterra, v. 144, n. 1, p. 85-94, 1999.

LUDLOW, M .M.; MUCHOW, R. C. A critical evolution of traits for improving crop yields in water-limited environments. Advances in Agronomy, San Diego, v. 43, p. $107-153,1990$

MAAS, E. V. Salt tolerance of crops. Applied Agricultural Research, New York, v. 1, n. 1, p. 12-26, 1986.

MAGALHÃES, J. R. de. Nutrição mineral do alho. Informe Agropecuário, Belo Horizonte, v. 12, n. 142, p. $20-30,1986$

MANGAL, J. L.; SINGH, R. K.; YADAV,A. C.; LAL, S.; PANDEY, U. C. Evaluation of garlic cultivars for salinity tolerance. Journal of Horticultural Science, Ashford, v. 65, n. 6, p. $657-658,1990$.

MUELLER, S.; BIASI, J. Comportamento de cultivares de alho plantio de janeiro, no planalto catarinense. Pesquisa Agropecuária Brasileira, Brasília, v. 25, n. 5, p. 783-788, maio 1990

NOBLE, C. L.; ROGERS, M. E. Arguments for the use of physiological criteria for improving the salt tolerance in crops. Plant and Soil, Dordrecht, v. 146, n. 1/2, p. $99-107,1992$

RAO, S. A. Genetic basis of variation for salt tolerance in maize. Euphytica, Dordrecht, v. 108, n. 3, p. 145-150, 1999.

SHALHEVET, J.; MORRIS, G. H.; SCHROEDER, B. P. Root and shoot response to salinity in maize and soybean. Agronomy Journal, Madison, v. 87, n. 3, p. 512-516, 1995.

Pesq. agropec. bras., Brasília, v. 37, n. 2, p. 167-176, fev. 2002 
SILVA, J. L. O.; ALVARENGA, M. R. A. Efeitos do choque frio sobre algumas características agronômicas do alho Chonan. I. Características morfológicas. Pesquisa Agropecuária Brasileira, Brasília, v. 19, n. 11, p. 13531357, nov. 1984

SILVA, N.; OLIVEIRA, G. D.; VASCONCELOS, E. F. C. HAAG, H. P. Nutrição mineral de hortaliças: absorção dos nutrientes pela cultura do alho. Solo, Piracicaba, v. 62, n. 1, p. 6-17, 1970.
SINGH, S. B.;ABROL, I. P. Effect of exchangeable sodium percentage on growth, yield and chemical composition of onion and garlic. Journal of the Indian Society of Soil Science, New Delhi, v. 33, p. 358-361, 1985.

SULTANA, N.; IKEDA, T. Effect of $\mathrm{NaCl}$ salinity on photosynthesis and dry matter accumulation in developing rice grains. Environmental and Experimental Botany, Amsterdam, v. 42, n. 3, p. 211-220, 1999. 